\title{
All quiet on the neuronal front: NMDA receptor inhibition by prion protein
}

\author{
Andrew D. Steele \\ Division of Biology, California Institute of Technology, Pasadena, CA 91125
}

The normal function of the prion protein $(\operatorname{Pr} P)$ - the causative agent of mad cow or prion disease-has long remained out of reach. Deciphering PrP's function may help to unravel the complex chain of events triggered by $\operatorname{PrP}$ misfolding during prion disease. In this issue of the JCB, an exciting paper (Khosravani, H., Y. Zhang, S. Tsutsui, S. Hameed, C. Altier, J. Hamid, L. Chen, M. Villemaire, Z. Ali, F.R. Jirik, and G.W. Zamponi. 2008. J. Cell Biol. 181:551-565) connects diverse observations regarding PrP into a coherent framework whereby PrP dampens the activity of an N-methyl-D-aspartate (NMDA) receptor (NMDAR) subtype and reduces excitotoxic lesions. The findings of this study suggest that understanding the normal function of proteins associated with neurodegenerative disease may elucidate the molecular pathogenesis.

The difficulty in determining the mechanisms of neurodegenerative disease is confounded by a poor understanding of the normal functions of the proteins implicated in disease. The relationship between the normal and toxic activities of proteins such as $\alpha$-synuclein in Parkinson's disease or Tau and amyloid precursor protein in Alzheimer's disease is gaining attention (Gitler and Shorter, 2007). Prion diseases are caused by misfolding of PrP, a conserved glycoprotein tethered to cell membranes by a glycosylphosphatidylinositol anchor (Prusiner, 1998). Highly expressed in neurons, PrP is also present in many other cell types (Aguzzi and Heikenwalder, 2006). However, neurons are the only cells thought to be adversely affected by the misfolding of PrP during prion diseases, which range from Creutzfeldt-Jakob disease and fatal familial insomnia in humans to bovine spongiform encephalopathy and chronic wasting disease in ruminants. Despite recent major advances into the structure, detection, and amplification of prions (for review see Caughey and Baron, 2006), the pathway of prion-mediated neurodegeneration remains to be understood. Several investigators suggest that the study of PrP's normal function may shed light on the pathogenic sequelae of prion disease (for reviews see Caughey and Baron, 2006; Steele et al., 2007a).

Correspondence to Andrew D. Steele: andydsteele@alum.mit.edu
The study of the normal function of PrP has been hampered by one simple fact revealed in 1992 in a seminal study from Charles Weissmann's laboratory (Bueler et al., 1992): the PrP knockout mouse has no overt phenotype (nor, for that matter, does the PrP-null cow [Richt et al., 2006]). However, subsequent studies have uncovered a multitude of phenotypes in PrP knockout mice, many of which manifest upon physiological challenge (for review see Steele et al., 2007a). These phenotypes range from defects in stem cell maintenance (Steele et al., 2006; Zhang et al., 2006) to dental abnormalities (Schneider et al., 2007) to enhanced susceptibility to ischemia (McLennan et al., 2004) and seizure (Walz et al., 1999) and even resistance to viral infection (Thackray and Bujdoso, 2002). Because of the diverse cellular functions attributed to PrP, molecular characterization of the phenotypes of PrP knockout mice is sorely needed, and Khosravani et al. (see p. 551 of this issue) seize the torch. By using electrophysiology, pharmacology, cell death assays, and biochemistry, they reveal a new and exciting role for PrP in directly silencing NMDARs (Khosravani et al., 2008).

Khosravani et al. (2008) launch their investigation by revisiting the peculiar electrophysiological properties of PrP-null neurons, which were first documented more than a decade ago (Collinge et al., 1994). Using hippocampal slices, they observed increased action potentials and a lower stimulation threshold to induce action potentials in PrP knockouts. Next, the authors find that the NMDAR antagonist aminophosphonovaleric acid rescues the effect of PrP deletion on NMDAR excitation, pointing toward NMDARs as key mediators of this hyperexcitability phenotype. NMDARs are a subclass of ligand-gated excitatory glutamate receptors that control fast synaptic transmission. Because they are calcium permeable (in addition to sodium), NMDARs have key roles in mediating constructive events like plasticity involved in learning but also participate in destructive events such as damage after stroke (Villmann and Becker, 2007).

Further slice recording experiments by Khosravani et al. (2008) revealed that in the absence of magnesium, which blocks NMDARs at resting potential, PrP-null neurons show an exaggerated hyperexcitability, reaching a seizurelike state much more rapidly than control slices. Next, the authors measure miniature excitatory postsynaptic currents in dissociated cultures of hippocampal neurons. Compared with controls, PrP-null neurons exhibited currents that had larger amplitudes and lasted much longer in response to the focal application of NMDA. Importantly, the authors performed additional control experiments to 
A

PrP inhibiting NMDA channel opening
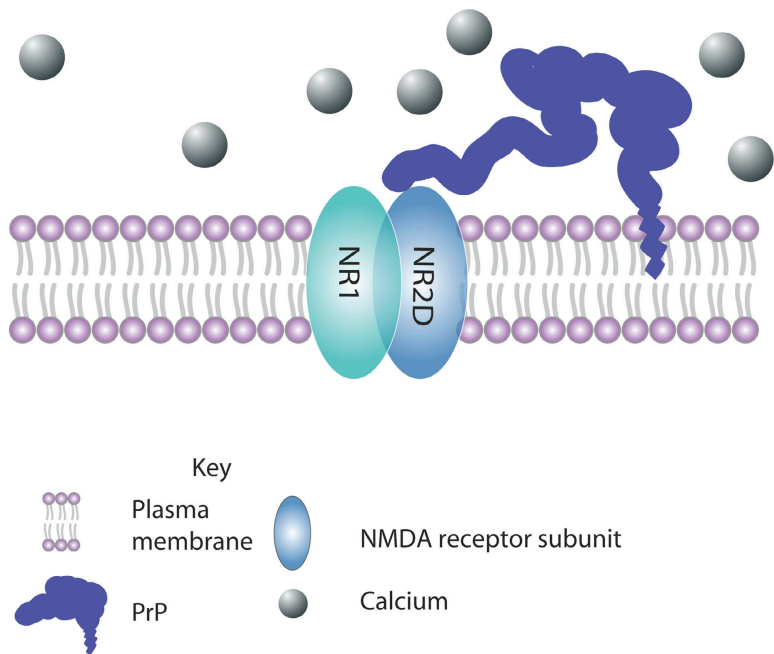

B Increased channel opening in absence of PrP

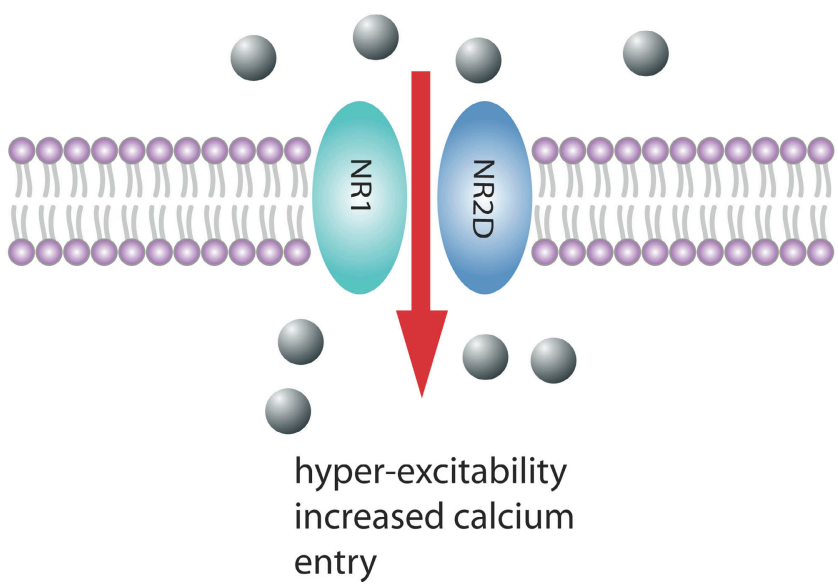

Figure 1. PrP may directly interact with the NR2D subunit of NMDAR to regulate its activity. (A) In wild-type neurons, PrP silences the NR2D-containing NDMAR, preventing depolarization and calcium entry. (B) In PrP-null neurons, the NR2D-containing NMDAR opens much more readily, leading to excessive calcium entry and more severe excitotoxic injury during conditions of excessive glutamate release. Magnesium, which blocks NMDAR at resting potential, is not depicted.

rule out potential artifacts caused by the use of PrP knockout neurons derived from a mouse strain of mixed genetic background by (1) using siRNAs to knock down PrP and (2) by reconstituting PrP into PrP-null neurons. To address the question of how PrP influences the NMDAR, Khosravani et al. (2008) go on to determine that PrP may interact directly with the NR2D subunit of NMDAR (Fig. 1) by demonstrating that PrP colocalizes with NR2D on the neuronal surface by immunofluoresence microscopy and that the two proteins can coimmunoprecipitate.

The next thrust of this story links PrP deletion to neurotoxicity mediated by excessive excitatory amino acid exposure, or excitotoxicity. Brief exposures of cultured neurons to NMDA lead to a marked increase in the death of PrP-null neurons compared with controls. The authors also show that in vivo application of NMDA directly into the hippocampus causes much larger lesions in PrP knockout brains. Thus, PrP protects against direct excitotoxic lesions, which provides a mechanistic understanding of a previous observation that PrP knockouts have increased susceptibility to stroke (McLennan et al., 2004). In conclusion, Khosravani et al. (2008) bridge the gap between PrP dampening excessive depolarization events in neurons to an important, biomedically relevant phenotype of PrP knockout mice.

This work raises many exciting questions about PrP's normal function, its relation to prion diseases, and the study of NMDAR activity. First, how much explanatory power does NMDAR inhibition provide in terms of illuminating other PrP knockout phenotypes (for reviews see Sakudo et al., 2006; Steele et al., 2007a)? Recent work from another laboratory has implicated kainite receptors, a glutamate-responsive ion-gated channel subtype, in mediating the enhanced damage of $\operatorname{PrP}$ knockout neurons in response to seizures (Rangel et al., 2007). Further experiments will be required to discern whether the results of Khosravani et al. (2008) and this seizure study are directly or indirectly related.
How do these new findings relate to prion diseases? Clearly, the detection of exaggerated NMDAR activity in prion-diseased samples would be a smoking gun implicating a loss of PrP function in prion disease. Do the familial mutants of PrP fail to effectively silence NMDARs, leading to hyperexcitability and a mechanism of neuronal damage similar to excitotoxicity? The cell death pathways involved in prion disease are far from understood (Steele et al., 2007b), and this new angle of investigation deserves attention, as perhaps NMDAR inhibition will have potential as a prion disease therapeutic strategy. Based on an interaction of PrP with NMDARs, one might speculate that the psychiatric symptoms of prion diseases could relate to defects in glutamatergic neurotransmission brought about either by PrP being titrated away from NR2D subunits or from direct interference by PrP oligomers or aggregates with NMDARs.

How does PrP silence NMDAR? As noted by Khosravani et al. (2008), PrP could block agonist binding, stabilize the closed state of the channel, or indirectly regulate function by interfering with signaling pathways affecting NR2D-containing NMDARs. With respect to NMDAR assembly, very little is known about NR2D subunits other than that they likely require NR1 subunits to reach the cell surface. In wild-type conditions, with ample PrP present on the neuronal cell surface, these channels will not open. What is the molecular logic of building a tonically inhibited NMDAR? Perhaps these channels only respond to extreme stimuli where they need not only a magnesium unblocking event but also a PrP-releasing event to open. The identification and characterization of additional interacting partners of PrP or NMDARs will be a complex and stimulating area of research. These questions aside, it is exciting to see the pieces of the PrP function puzzle start to come together.

A.D. Steele is supported by the Broad Fellows in Brain Circuitry program at the California Institute of Technology. 
Submitted: 27 March 2008

Accepted: 1 April 2008

\section{References}

Aguzzi, A., and M. Heikenwalder. 2006. Pathogenesis of prion diseases: current status and future outlook. Nat. Rev. Microbiol. 4:765-775.

Bueler, H., M. Fischer, Y. Lang, H. Bluethmann, H.P. Lipp, S.J. DeArmond, S.B. Prusiner, M. Aguet, and C. Weissmann. 1992. Normal development and behaviour of mice lacking the neuronal cell-surface PrP protein. Nature. 356:577-582.

Caughey, B., and G.S. Baron. 2006. Prions and their partners in crime. Nature. 443:803-810.

Collinge, J., M.A. Whittington, K.C. Sidle, C.J. Smith, M.S. Palmer, A.R. Clarke, and J.G. Jefferys. 1994. Prion protein is necessary for normal synaptic function. Nature. 370:295-297.

Gitler, A.D., and J. Shorter. 2007. Prime time for alpha-synuclein. J. Neurosci. 27:2433-2434.

Khosravani, H., Y. Zhang, S. Tsutsui, S. Hameed, C. Altier, J. Hamid, L. Chen, M. Villemaire, Z. Ali, F.R. Jirik, and G.W. Zamponi. 2008. Prion protein attenuates excitotoxicity by inhibiting NMDA receptors. J. Cell Biol. 181:551-565.

McLennan, N.F., P.M. Brennan, A. McNeill, I. Davies, A. Fotheringham, K.A. Rennison, D. Ritchie, F. Brannan, M.W. Head, J.W. Ironside, et al. 2004. Prion protein accumulation and neuroprotection in hypoxic brain damage. Am. J. Pathol. 165:227-235.

Prusiner, S.B. 1998. Prions. Proc. Natl. Acad. Sci. USA. 95:13363-13383.

Rangel, A., F. Burgaya, R. Gavin, E. Soriano, A. Aguzzi, and J.A. Del Rio. 2007. Enhanced susceptibility of Prnp-deficient mice to kainate-induced seizures, neuronal apoptosis, and death: role of AMPA/kainate receptors. J. Neurosci. Res. 85:2741-2755.

Richt, J.A., P. Kasinathan, A.N. Hamir, J. Castilla, T. Sathiyaseelan, F. Vargas, J. Sathiyaseelan, H. Wu, H. Matsushita, J. Koster, et al. 2006. Production of cattle lacking prion protein. Nat. Biotechnol. 25:132-138.

Sakudo, A., T. Onodera, Y. Suganuma, T. Kobayashi, K. Saeki, and K. Ikuta. 2006. Recent advances in clarifying prion protein functions using knockout mice and derived cell lines. Mini Rev. Med. Chem. 6:589-601.

Schneider, K., Y. Korkmaz, K. Addicks, H. Lang, and W.H. Raab. 2007. Prion protein (PrP) in human teeth: an unprecedented pointer to PrP's function. J. Endod. 33:110-113.

Steele, A.D., J.G. Emsley, P.H. Ozdinler, S. Lindquist, and J.D. Macklis. 2006 Prion protein $(\mathrm{PrPc})$ positively regulates neural precursor proliferation during developmental and adult mammalian neurogenesis. Proc. Natl. Acad. Sci. USA. 103:3416-3421.

Steele, A.D., S. Lindquist, and A. Aguzzi. 2007a. The prion protein knockout mouse: a phenotype under challenge. Prion. 1:83-93.

Steele, A.D., O.D. King, W.S. Jackson, C.A. Hetz, A.W. Borkowski, P. Thielen, R. Wollmann, and S. Lindquist. 2007b. Diminishing apoptosis by deletion of Bax or overexpression of $\mathrm{Bcl}-2$ does not protect against infectious prion toxicity in vivo. J. Neurosci. 27:13022-13027.

Thackray, A.M., and R. Bujdoso. 2002. PrP(c) expression influences the establishment of herpes simplex virus type 1 latency. J. Virol. 76:2498-2509.

Villmann, C., and C.M. Becker. 2007. On the hypes and falls in neuroprotection: targeting the NMDA receptor. Neuroscientist. 13:594-615.

Walz, R., O.B. Amaral, I.C. Rockenbach, R. Roesler, I. Izquierdo, E.A. Cavalheiro, V.R. Martins, and R.R. Brentani. 1999. Increased sensitivity to seizures in mice lacking cellular prion protein. Epilepsia. 40:1679-1682.

Zhang, C.C., A.D. Steele, S. Lindquist, and H.F. Lodish. 2006. Prion protein is expressed on long-term repopulating hematopoietic stem cells and is important for their self-renewal. Proc. Natl. Acad. Sci. USA. 103:2184-2189. 